STEM, STEAM, STREAM:

Posibilidades, reflexiones y experiencias

\title{
Propuesta formativa en STREAM: Una aproximación a la perspectiva global desde Cataluña
}

Recepción: 27/11/2020 | Revisión: 11/04/2021 | Aceptación: 05/08/2021 | Publicación: 01/10/2021

\section{Carles LINDÍN}

Universitat de Barcelona

carles.lindin@ub.edu

https://orcid.org/oooo-0002-3640-1258

(iD) Yuly VANEGAS

Universitat de Lleida

yuly.vanegas@udl.cat

https://orcid.org/oooo-0002-8365-1460

iD Antonio BARTOLOMÉ

Universitat de Barcelona

abartolome@ub.edu

https://orcid.org/oooo-00o2-8096-8278

\section{Laia COMA}

Universitat de Barcelona

lcomaq@ub.edu

https://orcid.org/oooo-0003-4501-840X

iD Carolina MARTÍN-PIÑOL

Universitat de Barcelona

carolinamartin@ub.edu

http://orcid.org/oooo-0002-0279-3839

Resumen: Fruto de la participación en el proyecto de investigación Standardization of STEM and Coding Trainings, dentro del programa Erasmus+, analizamos la situación de las STREAM en Cataluña, atendiendo a las acciones de apoyo, fomento e impulso que se desarrollan desde la administración. A partir del conocimiento de la realidad local y atendiendo a las necesidades educativas en un contexto global en el cual se inscribe la investigación, apuntamos la adecuación de utilizar el modelo $5 \mathrm{E}$, tanto como estrategia para el diseño de acciones formativas STREAM como para facilitar al alumnado el desarrollo de las actividades. A partir del trabajo de campo internacional de carácter cualitativo, basado en la combinación de grupos de expertos, estudio de casos y análisis de buenas prácticas, proponemos un plan de formación para el profesorado en materia STREAM $y$, en base a este, presentamos tres actividades, que corresponden a los niveles educativos de educación infantil, primaria y secundaria.

Palabras clave: STREAM; modelo 5E; educación infantil; educación primaria; educación secundaria. 
TRAINING PROPOSAL IN STREAM: AN APPROACH TO THE GLOBAL PERSPECTIVE FROM CATALONIA

Abstract: As a result of the participation in the research project Standardization of STEM and Coding Trainings, within the Erasmus+ program, we analyze the situation of STREAM in Catalonia, considering the support actions, promotion and impulse developed from the administration. Based on the knowledge of the local reality and attending to the educational needs in a global context in which the research is inscribed, we point out the adaptation of using the $5 E$ model, both as a strategy for the design of STREAM training actions and so as to facilitate the students development of activities. On the basis of qualitative international fieldwork, based on a combination of expert groups, case studies and analysis of best practices, we propose a training plan for teachers in STREAM and, from this, we propose three activities, which correspond to the educational levels of early childhood, elementary and secondary education.

Keywords: STREAM; 5 E model; early childhood education; primary education; secondary education.

\section{PROPOSTA FORMATIVA EN STREAM:}

\section{UNA APROXIMACIÓ A LA PERSPECTIVA GLOBAL DES DE CATALUNYA}

Resum: Fruit de la participació en el projecte d'investigació Standardization of STEM and Coding Trainings, dins del programa Erasmus+, analitzem la situació de les STREAM a Catalunya, fent atenció a les accions de suport, foment i impuls que es desenvolupen des de l'administració. A partir del coneixement de la realitat local $i$ tenint en compte les necessitats educatives en un context global en el qual s'inscriu la investigació, apuntem la idoneïtat d'utilitzar el model $5 E$, tant com a estratègia per al disseny d'accions formatives STREAM com per facilitar a l'alumnat el desenvolupament de les activitats. A partir del treball de camp internacional de caràcter qualitatiu, basat en la combinació de grups d'experts, estudi de casos $i$ anàlisis de bones pràctiques, proposem un pla de formació per al professorat en matèria STREAM i, en base a aquest, presentem tres activitats, que corresponen als nivells educatius d'educació infantil, primària i secundària.

Paraules clau: STREAM; model 5E; educació infantil; educació primària; educació secundària.

\section{Introducción}

En el presente artículo mostramos una propuesta de formación y de intervención en las aulas basada en las STREAM adecuada a la realidad curricular de Cataluña (Ley 12/2009), siguiendo las directrices escolares españolas (Ley orgánica 2/2006 y Ley orgánica 8/2013) y europeas (Recomendación 2006/962/CE), en el ámbito del trabajo sobre las competencias digitales en las diversas etapas de la educación obligatoria. Se trata de formulaciones que surgen del aprendizaje desarrollado durante la ejecución del proyecto de investigación Standardization of STEM and Coding Trainings (programa Erasmus+ Key Action KA2 - Cooperation for innovation and the exchange of good practices, Action Type KA201 - Strategic partnerships for school education). Un proyecto liderado por el Ministerio de Educación turco, en el cual ha participado la Universidad de Barcelona en colaboración con otras universidades (EUN Partnership AISBL, Bélgica; Gazi Üniversitesi, Turquía, y Spoleczna Akademia Nauk, Polonia).

Conocer y analizar la realidad europea sobre la implantación de las STREAM nos ha permitido realizar una propuesta formativa para docentes en materia STREAM y unas actividades de aprendizaje desde una perspectiva global, si bien aterrizadas a la realidad local. Por tanto, con la voluntad 
de incorporar el conocimiento y pensamiento crítico desde una perspectiva glocal (Murga-Menoyo y Novo, 2017). Tanto la formación docente como las actividades planteadas parten del modelo 5E, que establece un proyecto de enseñanza en cinco fases: involucrar (Engage, en inglés), explorar, explicar, elaborar y evaluar.

Ahora bien, es imprescindible situar las actividades en relación con la realidad más cercana en que pudieran ser implementadas. En este sentido, incorporamos una aproximación a la situación de las STREAM en el ámbito educativo en Cataluña.

Es de especial interés apuntar que las tres actividades propuestas se han seleccionado con la voluntad de ofrecer a la comunidad educativa una mayor diversidad. Por esta razón responden a tres niveles educativos (infantil, primaria y secundaria), así como a diversos ámbitos STREAM. De tal manera que se pueda constatar la adecuación del modelo $5 \mathrm{E}$ a las diversas edades del alumnado, a la vez que ofrecemos casos asumibles y realizables desde la perspectiva de plurales didácticas específicas.

Como resultado, reflexionaremos alrededor de las competencias que debieran ser adquiridas en la realidad digital del siglo XXI en el marco de las STREAM.

El objetivo de las STREAM de trabajar en el aula conjuntamente desde diversas disciplinas, especialmente poniéndolas en relación con la tecnología (por ende, la tecnología digital), pone en contacto esta tipología de propuestas con la adquisición de las competencias digitales intrínsecas a nuestros currículums escolares.

La competencia digital es incorporada como una competencia transversal en primaria y secundaria. Durante estas dos etapas, se consolida desde el trabajo en unas mismas dimensiones (instrumentos y aplicaciones; tratamiento de la información y organización de los entornos de trabajo y aprendizaje; comunicación interpersonal y colaboración; [ciudadanía,] hábitos, civismo e identidad digital). En la etapa de educación infantil se puede incorporar su tratamiento desde las diferentes áreas (descubrimiento de uno mismo y de los otros; descubrimiento del entorno; comunicación y lenguajes).

Aunque la competencia digital debiera ser incorporada en las distintas áreas temáticas, el hecho de no disponer de un área temática propia, por lo tanto, a falta de una asignatura y de un espacio-tiempo predeterminado, a menudo se convierte en un ámbito competencial poco incorporado en las aulas desde algunas didácticas específicas.

\section{Aproximación a las STREAM en Cataluña, desde la administración}

Tomamos el término 'STREAM' de forma general a lo largo del artículo, como nomenclatura genérica para referirnos a la combinación de las propuestas educativas que involucran (en inglés): Science, Technology, Reading, Engineering, Art y Math. La interpretamos como evolución de lo que en un inicio se llamó STEM y posteriormente se amplió a STEAM, hasta llegar al actual STREAM. Siendo conscientes de que algunos de los proyectos referenciados son STEM en sentido estricto, los entendemos como parte de la misma tipología de proyectos. En realidad, estamos ante la evolución del 
modelo STEM (de carácter profundamente científico-tecnológico), que a lo largo del tiempo se ha ampliado hacia realidades más cercanas al ámbito de las humanidades y las artes. En este sentido, con la evolución al modelo STREAM se constata como el pensamiento científico, tecnológico y matemático dialoga con el artístico y la lectura y la escritura, especialmente en los ámbitos de las artes digitales y las humanidades digitales.

La propuesta educativa que presentamos se enmarca en un entorno en el cual tanto las administraciones como los centros educativos promueven de forma activa la incorporación de las STREAM en las aulas.

Desde la administración autonómica catalana se constituye el Plan STEAMcat (Generalitat de Catalunya, 2017) siguiendo el Acuerdo GOV/19/2017, como respuesta al desequilibrio en la demanda laboral en los sectores relacionados con las STREAM. Incluso se apunta la necesidad del trabajo en colaboración con las empresas del sector para promover las vocaciones desde el ámbito educativo.

Este Plan desarrolla el Programa de innovación pedagógica STEAMcat, dentro de la línea de actualización científica y didáctica en relación con la formación general del profesorado, con el objetivo de:

- Mejorar los conocimientos en ciencia y tecnología de los profesores y su didáctica para generar mejores aprendizajes e interés por las STEM.

- Incrementar la motivación hacia las STEM mediante actuaciones formativas diversas, metodologías y recursos innovadores que las hagan más atractivas.

- Mejorar el conocimiento sobre estudios y profesiones STEM con acciones específicas de orientación educativas que incrementen las opciones STEM, los alumnos en general y de determinados colectivos. (Resolución ENS/1769/2018)

Con la voluntad de generar interés por los ámbitos trabajados y así potenciar vocaciones futuras en perfiles laborales que de otra manera serían muy distantes, se promueve el programa STEAMcat. Dirigido al alumnado de educación infantil, educación primaria y educación secundaria obligatoria, especialmente entre las estudiantes del sistema educativo y en contexto social desfavorecido.

Asimismo, incide en tres líneas de trabajo principales:

1. Sobre las metodologías y los recursos para la enseñanza y el aprendizaje.

2. Sobre la gestión y la organización educativa.

3. Sobre la importancia de la relación con la comunidad educativa.

En la actualidad, la incorporación de proyectos STREAM en las aulas catalanas se articula como un programa de innovación pedagógico. Por tanto, no es de implantación general, sino que está supeditado a la voluntad de los centros, siempre que participen de unos requisitos y compromisos establecidos por la administración (presentación de un proyecto, compromiso del centro...).

En realidad, más que de un currículum STREAM específico, podemos hablar de una mirada STREAM al currículum existente, en el que se fomenta especialmente el pensamiento científico, matemático y creativo. Por lo tanto, trata de trabajar las competencias del currículum desde diversas áreas de forma transversal, incluso estableciendo alianzas entre centros. 
Precisamente, ante esta necesidad y voluntad de incorporar las STREAM en las aulas, presentamos tres actividades de ámbitos de conocimiento diversos y de diferentes etapas educativas. Para que puedan ser incorporadas fácilmente en el aula, por parte de profesorado o equipos docentes, sin necesidad de participar en un proyecto de mayor envergadura. Asimismo, estas actividades se organizan siguiendo la metodología 5E, ya que se integra perfectamente en la filosofía STREAM, a la vez que proporciona una metodología útil al profesorado (para trabajar sobre nuevas actividades) y al estudiantado (estructura la adquisición de aprendizaje).

En línea con STEAMcat (Generalitat de Catalunya, 2021), presentamos una metodología replicable (el modelo 5E), que será aplicada a propuestas STREAM, que ofrecen a la vez recursos para la enseñanza y el aprendizaje (las actividades). Se encuadran dentro de las directrices del programa en cuanto a metodologías didácticas, que deberán ser significativas y contextualizadas, críticas, activas, centradas en el alumnado, sostenibles, inclusivas e integradoras, así como aunar el trabajo individual con el cooperativo y colaborativo (Generalitat de Catalunya, 2018).

Las tres actividades STREAM que presentamos ponen de manifiesto tanto la importancia como la incidencia de la ciencia y la tecnología en las propuestas de aprendizaje. Por tanto, a menudo la integración del trabajo sobre competencias del ámbito digital del alumnado de forma transversal.

Incluso el Parlamento Europeo enumera la competencia digital entre las competencias clave para el aprendizaje permanente, que son:

[...] una combinación de conocimientos, capacidades y actitudes adecuadas al contexto. Las competencias clave son aquellas que todas las personas precisan para su realización y desarrollo personal, así como para la ciudadanía activa, la inclusión social y el empleo. (Recomendación 2006/962/CE, p. 13)

Para su incorporación en el currículum, la administración catalana toma en consideración las conclusiones de la XXII Jornada de reflexión del Consejo Escolar de Cataluña (Generalitat de Catalunya, 2013), entre las que destaca la necesidad de incorporar las tecnologías digitales en todos los niveles educativos y ámbitos curriculares.

Por otro lado, Medina y Ballano (2015) se plantean retos alrededor de la cuestión de la transversalidad y el sentido de la tecnología digital (asignatura o competencia transversal, tecnología para el cambio de la práctica educativa).

De tal manera que trabajar desde la perspectiva STREAM y mediante el modelo 5E se acontece como una buena estrategia para realmente transversalizar la introducción y el aprendizaje sobre competencias digitales, en tanto que se relaciona con otras materias, con el entorno, y se trabajan las áreas y dimensiones competenciales en los diversos niveles (infantil, primaria y secundaria) que apuntábamos al inicio del artículo. 


\section{Propuesta formativa STREAM}

\subsection{Hacia un programa de formación}

Como hemos visto, la aproximación STREAM se basa en diversas estrategias para responder a los retos de los planteamientos actuales de las aulas, especialmente la incorporación de lo científico y lo tecnológico de forma transversal e interrelacionada entre ámbitos de conocimiento. Para potenciar la resolución de problemas reales con el objetivo principal de aportar una visión positiva/asertiva hacia las propuestas interdisciplinares e integradas en diversos ámbitos, hemos diseñado diversas propuestas formativas específicas para el profesorado entre las que encontramos la denominada Use of Educational Technology Teacher Trainer.

Es evidente que el objetivo final que se busca en dicho planteamiento es que el alumnado asimile que indagar para solventar las problemáticas derivadas de retos nuevos con sus conocimientos puede plantear soluciones aplicables a su vida diaria. Pero antes de fijarnos en este objetivo final, hace falta incidir en la necesidad de que el profesorado que imparte estas materias esté correctamente formado. De esta necesidad nace el planteamiento de esta parte del proyecto y es por ello que las tres actividades STREAM se han conceptualizado y planteado como una propuesta para el aula, que forma parte de un grupo de actividades para realizar una vez el profesorado ha sido formado, de forma ideal. Esto no obsta para que puedan ser realizadas al margen de un proceso de formación específico.

El modelo de formación del profesorado que proponemos responde principalmente a una alfabetización tecnológica y científica de todos sus participantes, así como de una adaptabilidad para los retos sociales de un contexto como el presente.

Las ideas principales para poder desarrollar dicha actividad formativa son aplicables al resto. Es obvio que algunas de ellas son básicas y garantizan la solvencia de la metodología planteada, nos referimos a elementos como disponer de ordenadores, tabletas o conexión a internet para cada uno de los participantes. Asimismo, es fundamental en este tipo de propuestas que los participantes puedan comunicarse de forma efectiva, para ello la actividad de formación del profesorado, de 36 horas, cuenta con más de una treintena de objetivos específicos para trabajar.

Señalamos las ideas generales bajo las que se conceptualizó. Destacamos que se buscaba principalmente formar al profesorado para que pueda desarrollar procesos de enseñanza y aprendizaje incorporando equipos informáticos usuales, así como software y hardware más específico (como los usados en las pizarras interactivas o los dispositivos móviles). Asimismo, se buscaba proporcionar la orientación y potenciar competencias profesionales del profesorado para adecuarlas a las metodologías de los ámbitos científicos y tecnológicos. A la vez, se pretendía dotarle de herramientas para aumentar las vocaciones científicas entre el alumnado a través de su orientación y desarrollo en el aprendizaje de STREAM.

En definitiva, se trata de dar a conocer estrategias para potenciar la creatividad y la innovación, que también conlleva tener conocimientos sobre el uso de los dispositivos móviles, de los recursos de un entorno virtual de aprendizaje (EVA) y de su aplicación en el contexto educativo. También se tiene en cuenta formar al profesorado con los criterios fundamentales acerca de la orientación en la selección de contenidos y materiales electrónicos para sus estudiantes del futuro. 


\subsection{El modelo 5E}

Tal y como se ha apuntado, para realizar las actividades que forman parte del proceso de formación del profesorado, seguimos el modelo de enseñanza y aprendizaje 5E (Tabla 1), estructurado en cinco etapas (involucrar, explorar, explicar, elaborar y evaluar), que tiene su origen en la Biological Sciencies Curriculum Study (BSCS) (Bybee y Landes, 1990). Desde una perspectiva constructivista, surge de la combinación de diversos métodos instruccionales (Bastida-Bastida, 2019), que permite centrar la atención del alumnado (Olimid, 2019). Parten de la necesidad de que la juventud aprenda "acerca de la ciencia, la tecnología y la salud, ya que necesitan comprenderlos y usarlos en su vida diaria y como futuros ciudadanos" (Bybee y Landes, 1990, p. 92).

Esta propuesta tiene la voluntad de incorporar la tecnología (en algún caso las TIC) con el objetivo de fomentar en el alumnado las habilidades científicas y digitales en combinación con las propias de las materias. Al mismo tiempo, pretende fomentar las vocaciones científicas e incorporar los ámbitos de conocimiento de las ciencias sociales y las humanidades a la realidad digital. De esta manera, el alumnado se acerca a una metodología de aprendizaje y de trabajo planificado y estructurado que asimila como útil y favorable a su aprendizaje desde diversos puntos de vista:

- Permite conectar los saberes científicos-humanísticos mediante una metodología adecuada con independencia del ámbito temático.

- Fomenta el trabajo sobre las competencias digitales de forma transversal.

- Ofrece un modelo pedagógico al profesorado que puede ser aplicado a multitud de casos una vez se ha realizado una primera aproximación a su manejo.

\begin{tabular}{|c|c|}
\hline Etapas & Explicación \\
\hline Involucrar & $\begin{array}{l}\text { El instructor busca conocer al alumnado, comprender el nivel de sus } \\
\text { conocimientos e identificar los aspectos fuertes y débiles de su formación } \\
\text { educativa. Esta fase también se ocupa de los aspectos motivacionales, con el } \\
\text { objetivo de involucrar al alumnado y hacer que le guste aprender. }\end{array}$ \\
\hline Explorar & $\begin{array}{l}\text { El alumnado explora nuevos conceptos mediante la lectura, la experimentación o } \\
\text { las discusiones. Los conceptos no se han presentado ni explicado directamente } \\
\text { antes. Con este enfoque, el alumnado aumenta activamente sus conocimientos. El } \\
\text { instructor responde preguntas o da instrucciones, pero no transmite directamente } \\
\text { el conocimiento de manera tradicional. }\end{array}$ \\
\hline Explicar & $\begin{array}{l}\text { El instructor presenta las definiciones formales de conceptos. Esta fase se enfoca } \\
\text { en aspectos particulares y generalmente es una consecuencia de la fase de } \\
\text { exploración, donde el alumnado debe utilizar los conocimientos adquiridos durante } \\
\text { la fase de exploración. Se le invita a hacer preguntas y aclarar sus conceptos. }\end{array}$ \\
\hline Elaborar & $\begin{array}{l}\text { El alumnado desarrolla una comprensión más profunda y amplia al practicar lo } \\
\text { que ya ha aprendido. Se supone que se deben realizar nuevos experimentos, } \\
\text { crear presentaciones o explicar conceptos a sus compañeros de estudios. }\end{array}$ \\
\hline Evaluar & $\begin{array}{l}\text { El alumnado es evaluado por métodos formales o informales, como exámenes, } \\
\text { cuestionarios, trabajos escritos, proyectos y carpetas de trabajo. Esta fase permite } \\
\text { al instructor estar al tanto de su progreso y tomar las acciones correspondientes. }\end{array}$ \\
\hline
\end{tabular}

Tabla 1. Etapas del modelo 5E. Fuente: Olimid (2019). 
En definitiva, para el profesorado, el modelo 5E aporta una estrategia interesante en favor del aprendizaje significativo del alumnado (Bastida-Bastida, 2019), en que se incorporan las prácticas de indagación (Ballone y Duran, 2004). Es más, se trata de una metodología que llega a afectar positivamente en las actitudes del alumnado, además de incidir en la mejora del rendimiento académico.

\section{Propuesta de actividades STREAM}

Si bien es cierto que durante el proyecto de investigación origen del presente artículo se focalizó en STEM, para este trabajo hemos creído interesante ampliar el concepto a STREAM y, por tanto, ofrecer actividades que corresponden a diferentes niveles educativos y que incluyen un mayor número de ámbitos de conocimiento. Se trata de actividades diseñadas por los investigadores con el objetivo de promover las competencias STREAM en las aulas de infantil, primaria y secundaria.

El aprendizaje STREAM permite al alumnado de diferentes niveles educativos explorar, construir y compartir todo aquello que conoce y descubre de su entorno, a la vez que promueve vocaciones científicas. Se trata de una educación que se caracteriza por:

- Incorporar un enfoque integrado en el que diferentes áreas de aprendizaje se relacionan.

- Desarrollar el interés por el ámbito científico desde edades tempranas.

- Considerar que la resolución de problemas y el enfrentar y comprender situaciones de la vida cotidiana son motores para el diseño y la implementación de actividades.

- Experimentar como proceso natural y central en el fomento de una actitud indagadora.

- Incorporar la tecnología digital como una estrategia/herramienta más para el conocimiento y la actuación sobre el entorno.

Desde el punto de vista metodológico, las actividades propuestas son fruto de un trabajo de campo previo de carácter cualitativo, basado en técnicas tales como: los grupos de expertos (en materia de educación tecnológica competencial y STREAM); el estudio de casos, mediante el análisis y observación en el aula de actividades STREAM, y el análisis de buenas prácticas. Dichas técnicas se han desarrollado en distintos escenarios geográficos, que responden al carácter internacional del proyecto (Turquía, Bélgica, Polonia y España).

Con el grupo de expertos tratamos necesidades formativas de los estudiantes (por ende, del profesorado) atendiendo a las realidades que se plantean en cada entorno. Asimismo, hemos analizado diversas propuestas gubernamentales, atendiendo a los factores que promueven la incorporación de las STREAM en el aula.

Hemos seleccionado y analizado buenas prácticas en los diversos territorios, atendiendo tanto a propuestas del ámbito educativo formal como a iniciativas del sector privado, que promueven y dan soporte a la introducción del pensamiento tecnológico y computacional en la escuela.

Finalmente, hemos realizado la observación en el aula en diferentes escuelas, tanto de proyectos STREAM como en acciones de difusión del componente científico, tecnológico y digital. 


\subsection{Propuesta para educación infantil: Puentes y escaleras para salvar vidas}

Esta actividad STREAM, diseñada para la segunda etapa de infantil (4-6 años), tiene como objetivo desarrollar habilidades creativas, constructivas y comunicativas relacionadas con hechos y conceptos cercanos a la realidad de los más pequeños. Se trabajan aspectos diversos de las áreas del currículum de educación infantil, como el descubrimiento del entorno y la comunicación y lenguajes. La actividad se estructura en las diversas fases ya expuestas en este artículo: el modelo 5E (involucrar, explorar, explicar, elaborar y evaluar). Plantea a los niños y las niñas el desafío de diseñar y construir diversos puentes con el objetivo de rescatar a un muñeco del fondo de un pozo y conseguir que llegue hasta su casa.

\section{a) Involucrar}

Para empezar, el profesor o la profesora debe plantear a su alumnado el problema que debe resolver, indicando el material determinado con el que cuenta y que debe ser resuelto de forma colaborativa y con la participación de todos. El reto o problema para resolver se basa en rescatar del fondo de un pozo a un muñeco construyendo las escaleras y los puentes necesarios para que pueda salir del pozo y llegue sano a su casa.

\section{b) Explorar}

En esta fase de exploración se presentan los materiales ante los niños y las niñas para que los analicen, observen, midan, etc. Concretamente los materiales son:

- Caramelos de goma de mascar (o bolas de plastilina)

- Palillos

- Palitos de paleta

- Muñeco pequeño (Lego o similar)

- Contenedores opcionales para proporcionar desafíos para rescatar al muñeco

- Piezas de madera o similar para construir la casa del muñeco.

A continuación, deben empezar a pensar cómo se pueden construir puentes o escaleras con los materiales de que disponen. Empieza aquí el ejercicio de creatividad e imaginación de los niños y las niñas.

\section{c) Explicar}

Según las edades de los niños y las niñas puede ser que requieran "ver" el concepto del problema planteado para entender la idea. En este caso, se podría visualizar, por ejemplo, un fragmento del episodio de Magic School Bus (NannieDaniel3502, 2018) en el que se muestra la construcción de puentes similares a los que ellos y ellas pueden diseñar.

En este caso, el profesor o la profesora debe pausar el video en aquellas escenas en las que se observe la construcción de puentes o estructuras similares a las que los niños y las niñas puedan necesitar diseñar para salvar a su muñeco del pozo. Este puede ser un buen momento también para hablar con ellos acerca de las formas observadas, cuántos caramelos de goma de mascar y palillos de dientes se necesitan, y cómo funcionaba el puente.

Más allá de la visualización de este video (o similar), que es opcional, es el momento de explicar con más detalle el reto o problema a resolver. Para ello, el profesor o la profesora coloca en 
el interior de un recipiente (que simula ser el pozo) el muñeco y, por otro lado, a cierta distancia, coloca unas piezas de construcción para simular la casa del muñeco. De este modo, los niños y las niñas ya observan cuál es el punto de inicio del experimento (el pozo) y cuál es el punto final al cual deben hacer llegar a su muñeco (la casa).

\section{d) Elaborar}

Ahora ha llegado el momento de dejar solos a los niños y las niñas para que experimenten con los materiales y piensen cómo construir las escaleras y puentes necesarios, cuántos, de qué dimensiones y longitud, etc.

Hay que dejarles tiempo suficiente para que prueben modelos distintos, que descubran por sí solos qué materiales son más adecuados para un tipo de construcción u otra, para que hagan y deshagan; para que hablen entre ellos y compartan sus puntos de vista y propuestas de acción, y para que debatan y lleguen a conclusiones y decisiones conjuntas para resolver el reto planteado.

\section{e) Evaluar}

En este tipo de actividades, la mejor evaluación es la comprobación del material diseñado, para corroborar si es el adecuado para resolver el reto inicialmente planteado. Así pues, una vez que las escaleras y los puentes están construidos, los niños y las niñas deben comprobar si están bien diseñados para que el muñeco pueda salir del contenedor (del pozo), e incluso ir de un lado a otro utilizando los puentes y pasarelas creadas, hasta llegar a la meta final, a la casa del muñeco.

Para ello, deben tomar aún las últimas decisiones: qué construcción poner a lo largo del recorrido pensando en sus características y las propias del lugar, es decir, si hay desnivel, si la distancia entre los recipientes (puntos de apoyo) son largas o cortas, qué obstáculos (desafíos intencionados) hay que resolver, etc.

Una vez estén colocadas las diferentes construcciones en el sitio elegido, es el momento de mover el muñeco a través de las escaleras y puentes diseñados y estratégicamente colocados para comprobar si puede o no llegar sin problemas hasta su casa. Si el muñeco logra salir del pozo y llegar hasta su casa pasando por las estructuras diseñadas, iel reto se ha conseguido!

Desde el punto de vista del profesor o la profesora, para evaluar el proceso y el trabajo desarrollado por los niños y las niñas, puede hacer una evaluación mediante la observación durante las fases anteriores, así como pedir en esta fase final que expliquen de forma oral cuál ha sido su experiencia, cómo han trabajado en grupo, qué decisiones han tomado, con qué dificultades se han encontrado y cómo las han solucionado.

Con esta actividad se busca:

- Aprender a trabajar en equipo.

- Descomponer y analizar el problema y la situación contextual del mismo para pensar las acciones necesarias con el objetivo de resolverlo.

- Tomar decisiones colectivamente e identificar múltiples soluciones para un mismo problema. 
- Desarrollar habilidades de construcción.

- Aplicar el pensamiento lógico y el razonamiento para la resolución de problemas.

- Experimentar acciones mediante el ensayo-error.

- Desarrollar la creatividad.

\subsection{Propuesta para educación primaria: El desafío del avión de papel}

A continuación, se describe una actividad para el ciclo superior de primaria. El objetivo de esta actividad es involucrar a los niños en procesos de resolución de problemas de situaciones del mundo real. Se busca promover la formulación de ideas científicas basadas en la experimentación, el razonamiento y la comunicación. También se espera que los alumnos apliquen y conecten aprendizajes de diferentes áreas: matemáticas (medición y geometría), ciencias (materiales y fuerza) y tecnología (diseño).

Para la descripción hemos aplicado el modelo 5E con sus cinco fases, que estimamos clave en el desarrollo de propuestas STREAM: involucrar, explorar, explicar, elaborar y evaluar.

La actividad plantea a los niños y las niñas el desafío de diseñar y elaborar un avión de papel de tal manera que permanezca en el aire tanto tiempo como sea posible.

\section{a) Involucrar}

En esta fase se debe motivar al alumnado introduciendo la actividad hablándole del desafío. También se deben comentar y explicar las instrucciones y condiciones que tener en cuenta, por ejemplo:

- El trabajo se desarrolla en grupo.

- Cada grupo debe proponer un diseño consensuado del avión.

- El avión se construye con una sola hoja de papel A4.

- Cada grupo puede hacer volar su avión. El avión se lanza a mano.

- Recordar que, para esta actividad, la distancia y la velocidad no son tan importantes como la duración del vuelo.

- Cada grupo cronometra el vuelo de su avión usando un cronómetro.

- Cada grupo tiene la oportunidad de rediseñar su avión y volver a ponerlo a volar para ver si aumenta el tiempo que permanece en el aire.

Se puede aprovechar también este momento para hablar con los niños y las niñas sobre la importancia de considerar algunas reglas de seguridad:

- Nunca arrojar un avión de papel a otra persona, animal u objeto que pueda ser dañado si lo golpea.

- Tener en cuenta que los aviones de papel pueden curvarse o cambiar de dirección después de ser lanzados, así que es importante asegurar que su área de vuelo esté despejada.

- Si la experimentación del vuelo de los aviones se realiza al aire libre, nunca vuele el avión cerca de automóviles en movimiento ni corra hacia la calle siguiendo el recorrido de su avión.

\section{b) Explorar}

Para continuar motivando y animando al alumnado en el proceso de diseño, se puede co- 
menzar leyendo conjuntamente el libro ilustrado El sueño de Engibear (King, 2013). En este libro se cuenta cómo Engibear, un ingeniero, sueña con varios diseños y comienza a construir un "robot de oso". Las primeras versiones del robot fallan, pero Engibear sigue intentándolo. El Bearbot Tipo 10 es el diseño final, que recompensa la persistencia de Engibear.

Después de la lectura, conviene discutir con el alumnado el proceso seguido por el protagonista de la historia en el diseño de su robot e invitarle luego a seguir estos pasos.

Se puede también hablar con los niños y las niñas sobre qué tipo de ingenieros o ingenieras pueden trabajar para una aerolínea y por qué. Por ejemplo, ingenieros/as aeroespaciales (diseñan aviones), ingenieros/as mecánicos/as (diseñan los motores), ingenieros/as químicos/as (determinan la combustión del combustible), ingenieros/as informáticos/as (diseñan sistemas de vuelo), etc.

Posteriormente se puede visualizar con la clase un vídeo de un avión despegando y aterrizando. Mientras miran el vídeo, el alumnado puede pensar en: a) el movimiento y la dirección del avión durante el despegue, el vuelo y el aterrizaje; b) la velocidad del avión, y c) la forma y las características del avión que le permiten volar.

En todos estos procesos es fundamental invitar al alumnado a registrar sus observaciones en sus libretas. Es el momento también de animarle a dibujar un esquema de un avión, distinguiendo en este, la nariz, la cola, las alas y el cuerpo.

\section{c) Explicar}

En esta fase se debe invitar a los niños y las niñas a comenzar con sus diseños, los cuales deben tener en cuenta todo lo discutido anteriormente. Es importante sugerirles que sigan un protocolo de trabajo. Es necesario insistir en esta fase que, cuando prueben sus aviones, pueden pensar en formas de mejorarlo, porque luego tienen la oportunidad de rediseñar y volver a probar. También es importante que se pida al alumnado que escriba una hoja informativa para otros compañeros explicando cómo hicieron su avión de papel y cómo voló.

\section{d) Elaborar}

En esta fase es primordial solicitar al alumnado que represente y etiquete las medidas de su diseño de avión antes de su construcción y prueba. Es clave recordar a los niños y las niñas que al experimentar el vuelo de los aviones es fundamental registrar el tiempo que su avión está en el aire, explicar cómo fue lanzado y describir lo que observan sobre su trayectoria de vuelo y la forma en que aterriza.

En este momento, el profesor o la profesora puede circular por el aula y tomar notas sobre las diferentes propuestas. Es el momento de hablar con los niños y las niñas sobre su diseño, la forma de las alas, los tamaños, los instrumentos que necesitan para medir, etc.

Después de probar sus aviones, cada grupo debe rediseñar su propuesta, pero, antes de rediseñar y volver a probar sus aviones, los grupos de estudiantes deben discutir y registrar cómo y por qué cambian tanto su método de lanzamiento como su diseño inicial. En este momento se puede conversar con el alumnado sobre las cuatro fuerzas asociadas con el vuelo, a saber: elevación, 
empuje, arrastre y peso.

\section{e) Evaluar}

Una manera de evaluar el trabajo desarrollado puede partir de la presentación oral de cada grupo a toda la clase. Solicitar al alumnado que describa el proceso seguido por el grupo, resaltando los puntos en los que tuvo más dificultades. También es clave animarle a valorar las similitudes y/o diferencias entre las propuestas. Finalmente, se le puede invitar a reflexionar sobre los conocimientos que considera que ha utilizado para responder al desafío.

Con esta actividad se busca:

- Desarrollar habilidades de investigación.

- Fomentar el trabajo colaborativo.

- Conocer características de los materiales (papel, cartón).

- Aprender a tomar decisiones basadas en el razonamiento.

- Aplicar el aprendizaje de las matemáticas (medición y geometría), la ciencia (fuerza) y la tecnología (diseño) para resolver problemas del mundo real.

- Usar procesos de resolución de problemas para apoyar la formulación de ideas científicas basadas en el razonamiento y la comunicación.

- Desarrollar la creatividad.

- Reconocer la importancia de conectar diferentes áreas (Ciencia, Matemáticas, Tecnología e Ingeniería) para comprender mejor diversas situaciones.

\subsection{Propuesta para educación secundaria: El laboratorio de la literatura}

El objetivo de esta actividad, diseñada para el alumnado que finaliza la educación secundaria (15-16 años), es que conozca que son las humanidades digitales, más concretamente el campo de conocimiento al que podemos llamar filología digital: en este caso, analizar el hecho literario con herramientas digitales. Así puede poner en relación las competencias propias del pensamiento científico y las habilidades tecnológicas con las del pensamiento humanístico y el análisis literario.

De esta manera, se enfrenta no solo a nuevas maneras de analizar la literatura, sino que también se sitúa ante nuevas posibilidades de plantearse preguntas y encontrar respuestas. En definitiva, incorporar las humanidades al ámbito digital desde un proyecto STREAM. En concreto, haciendo especial énfasis en cuatro aspectos: Technology, Reading, Art y Math.

La propuesta se presenta siguiendo el modelo 5E aplicado también en las actividades anteriores.

\section{a) Involucrar}

Para conocer al alumnado y sus conocimientos previos, se le pregunta sobre su experiencia de lectura literaria, con el objetivo de averiguar qué le interesa, qué le gusta/disgusta.

De este modo, podremos realizar una cosmovisión de la relación con el hecho artístico en general, y el literario en particular, de cada uno de los y las estudiantes de forma individual a la vez que del grupo clase. 
Seguidamente, se pone en común qué relación tienen con el análisis de la literatura. ¿Qué tipo de literatura analizan, desde qué perspectiva?

Podemos convenir que gran parte de las respuestas pueden coincidir en el hecho de que el espacio en que analizan la literatura es mayoritariamente en el aula escolar, a partir de lecturas obligatorias de corte académico. En este sentido, el acercamiento acostumbra a ser entre histórico (la importancia histórica de la obra y el/la autor/a en un contexto determinado) y biográfico (relación de los eventos vitales con el resultado de la obra artística). Si hay quien ha participado en grupos de lectura, se recurre a esta experiencia para ahondar en diversos puntos de vista de acceso y análisis/comentario literario.

Posteriormente, nos iniciamos en el método científico para trabajar sobre los textos, específicamente sobre el análisis de datos. Para motivar al alumnado, se le pregunta acerca del método científico. Después de saber su conocimiento sobre el tema, le preguntaremos si es posible analizar la literatura siguiendo el método científico, a partir del análisis de datos objetivos.

Es este punto apuntaremos la existencia de:

- Corpus lingüísticos y literarios.

- Posibilidades de análisis de estos corpus.

Se le pregunta sobre qué querría analizar si fuera posible, si tuviéramos herramientas digitales que lo permitieran.

En cuanto a la preparación de la actividad, se explica que el trabajo se realiza en grupo y que se da mayor importancia al proceso que al resultado, es decir, a la explicación de qué ha hecho, por qué, cómo... más que al resultado final del análisis literario.

\section{b) Explorar}

Con el objetivo de explorar nuevos conceptos, se invita al alumnado a que busque información sobre:

- Metodologías de análisis literario (con y sin tecnología).

- Estilometría y literatura.

- La diferencia entre Close Reading (o lectura cercana) y Distance Reading (o lectura distante).

Es posible repartir temas por grupos para que posteriormente los pongan en común.

El profesor o la profesora responde dudas concretas y da apoyo en la búsqueda de información. Si lo considera oportuno, puede facilitar el acceso a lecturas especializadas, por ejemplo: Martos y Martos (2018) o Boto (2018). El alumnado expone el resultado de los temas trabajados.

\section{c) Explicar}

El profesor o la profesora cohesiona y da hilo argumental a la explicación realizada por el alumnado, a la vez que la matiza y amplía, teniendo en cuenta el trabajo que se va a elaborar. 
Es el momento de explicar qué tipo de investigación/análisis se va a desarrollar y con qué herramienta. Como ejemplo, el profesor o la profesora propone el análisis sobre un texto literario que se encuentra digitalizado. A sabiendas de la multitud de bases de datos disponibles, recomendamos la selección de alguna obra del catálogo de la Biblioteca Virtual Miguel de Cervantes (http://www.cervantesvirtual.com/).

Se utiliza la herramienta gratuita y en línea Voyant Tools (https://voyant-tools.org/). Atendiendo que esta tipología de entornos puede evolucionar o desaparecer, apuntamos la diversidad de software gratuito disponible que podría ser utilizado en sustitución de Voyant Tools. Basta con realizar una búsqueda en internet con los conceptos "estilometría software". Asimismo, en el web Tools for Corpus Linguistics (https://corpus-analysis.com/) se recopilan centenares de herramientas clasificadas por objetivos, plataformas y coste.

Siguiendo con el texto a analizar, proponemos el ejemplo de El Conde de Lucanor. Se copian los enlaces de los diversos capítulos (puede trabajarse algún capítulo o distribuirlos por grupos) y se incorporan a Voyant Tools para ser analizados.

Se exploran los diferentes apartados del análisis, con especial énfasis en las dos ventanas de la zona derecha de la herramienta. En la superior podemos analizar las palabras más utilizadas. Una vez seleccionado el término que nos interese, si clicamos, en la ventana inmediatamente inferior observamos las concurrencias de esta palabra, es decir, todas sus apariciones en contexto. A modo de ejemplo, nos serviría para preguntarnos qué tipo de acciones realiza algún personaje, con qué significado se suele relacionar alguna palabra, incluso desde un punto de vista lingüístico, sobre el uso de preposiciones o construcciones.

\section{d) Elaborar}

El alumnado elabora su investigación y análisis. Indica:

- Preguntas de investigación.

- Objetivos de investigación.

- El proceso de análisis explicado paso a paso.

- Resultados obtenidos.

- Explicación de la experiencia.

\section{e) Evaluar}

El alumnado debe presentar en público cómo ha desarrollado la investigación/análisis. De manera que puede constatar cómo ante el análisis de un mismo texto se pueden plantear preguntas y objetivos de investigación diversos. Asimismo, las estrategias y los resultados también son plurales, de tal manera que se accede a un conocimiento diferente de la obra analizada a partir de las aportaciones de los grupos.

Se debe debatir sobre:

- Las diferencias entre los resultados de los distintos grupos.

- La utilidad del uso de herramientas digitales.

- Las posibilidades de analizar centenares de obras a la vez. ¿Qué preguntas y objetivos de investigación/análisis se pueden plantear? 
La evaluación se realiza a partir de tres elementos:

- El análisis realizado con herramientas digitales.

- La presentación sobre su análisis y las aportaciones al debate.

- Documento posterior breve (un folio) en que se ponga de relieve lo aprendido durante el proceso y los diversos puntos de vista y realidades observados en la fase de exposición de resultados y debate.

Con esta actividad se busca:

- Dar a conocer las posibilidades de las humanidades digitales y de la filología digital.

- Construir conocimiento sobre obras y autores literarios.

- Explorar y debatir las oportunidades de análisis de la "lectura distante".

- Proponer análisis innovadores como posibles nuevas lecturas.

- Saber buscar y seleccionar fuentes de información.

- Proponer investigaciones en el entorno escolar.

- Debatir sobre los resultados y oportunidades de uso en el conocimiento y análisis del patrimonio literario.

\section{Conclusiones}

La experiencia adquirida en el proyecto de investigación referido evidencia la necesidad de trabajar conjuntamente en entornos internacionales para promover proyectos de formación del profesorado que se ajusten a realidades locales desde perspectivas globales. De hecho, partiendo de estándares internacionales que permiten ser adecuados a las diversidades de los sistemas educativos y a sociedades concretas.

En la misma línea, conocer las propuestas en las aulas de diversos países europeos, así como proyectos destacados de incorporación de las STREAM en los diversos niveles educativos, posibilita acercarse a la realidad próxima desde una perspectiva contextualizada, que imprime una mirada abierta y transversal al currículum, más allá de las legislaciones educativas, de carácter siempre circunstancial. Precisamente este es el sentido primigenio de las STREAM: el trabajo interdisciplinar. Incluso diríamos, intercurricular.

Tanto la propuesta de formación del profesorado como las actividades para el aula aportan un ejemplo de incorporación de las STREAM como un camino de doble sentido. Por un lado, la propuesta de formación incide en el modelo 5E como estrategia metodológica, que es de utilidad al profesorado en su proceso formativo, pero que a la vez servirá al alumnado para incorporar una metodología de aprendizaje que le ponga en contacto con el mundo y con sus conocimientos previos. Por otro lado, la interdisciplinariedad intrínseca de las STREAM puede inducir al profesorado a ahondar en la incorporación de propuestas de aprendizaje conectadas entre materias, hecho que produce en el alumnado una visión amplificada de su formación, más allá de espacios de conocimientos estancos, desde una perspectiva claramente competencial e interdisciplinar.

En definitiva, propuestas a la vez formadoras e inspiradoras para el profesorado, sin perder de vista la adecuación para el aprendizaje del alumnado. 


\section{Agradecimientos}

Este trabajo se ha realizado como consecuencia del aprendizaje realizado durante el proyecto de investigación Standardization of STEM and Coding Trainings (programa Erasmus+ Key Action KA2 - Cooperation for innovation and the exchange of good practices, Action Type KA201 - Strategic partnerships for school education). Grant Agreement No. 2018-1-TR01-KA201-058663.

\section{Referencias}

Acuerdo GOV/19/2017, de 28 de febrero, por el que se crea el grupo de trabajo interdepartamental STEMcat, de las vocaciones científicas, tecnológicas, en ingeniería y matemáticas, DOGC 7320 (2017). https://bit.ly/3jjXOlG

Ballone, L. y Duran, E. (2004). The $5 \mathrm{E}$ instructional model: A learning cycle approach for inquiry-based science teaching. The Science Education Review, 32(2), 49-58. https://files.eric.ed.gov/fulltext/EJ1058007.pdf

Bastida-Bastida Izaguirre, D. (2019). Adaptación del modelo $5 \mathrm{E}$ con el uso de herramientas digitales para la educación: propuesta para el docente de ciencias. Revista Científica, 1(34), 73-80. https://doi.org/10.14483/23448350.13520

Bybee, R. W. y Landes, N. M. (1990). Science for life and living: An elementary school science program from biological sciences curriculum study. The American Biology Teacher, 52(2), 92-98. https://doi.org/10.2307/4449042

Boto, M. A. (2018). Mapa estilométrico de la narrativa de Eduardo Mendoza: aproximación a un análisis estilístico computacional de textos literarios. Epos: Revista de Filología, 33, 99-114. https://doi.org/10.5944/epos.33.2017.18281

Generalitat de Catalunya [Consell Escolar de Catalunya] (2013). L’impacte i la contribució de les tecnologies digitals en l'educació. XXII Jornada de reflexió, 10 de novembre de 2012: conclusions. http://hdl.handle.net/20.500.12694/505

Generalitat de Catalunya (2017). Pla STEMcat d’impuls de les vocacions científiques, tecnològiques, en enginyeria i en matemàtiques. https://bit.ly/2VoOLry

Generalitat de Catalunya [Departament d'Educació] (2018). Marc pedagògic del Programa STEAMcat. Recuperado de https://projectes.xtec.cat/steamcat/categoria/marc_pedagogic/

Generalitat de Catalunya [Departamento d'Educació] (2021). STEAMcat | Programa d'inovació STEAMcat. https://projectes.xtec.cat/steamcat/

King, A. (2013). Engibear's Dream. Little Steps Publishing.

Ley 12/2009, del 10 de julio, de educación, DOGC 5422 (2009). https://dogc.gencat.cat/es/document-del-dogc/?documentId=480169

Leyorgánica2/2006, de3 demayo, deEducación,BOE106(2006).https://www.boe.es/eli/es/lo/2006/05/03/2 Ley orgánica 8/2013, de 9 de diciembre, para la mejora de la calidad educativa, BOE 295 (2013). https://www.boe.es/eli/es/lo/2013/12/09/8

Martos, E. y Martos, A. (2018). Categorizaciones de la lectura y praxis cultural en la era digital: distant reading vs. close reading. Investigación Bibliotecológica, 32(74), 19-33.

https://doi.org/10.22201/iibi.24488321xe.2018.74.57904

Medina, A. y Ballano, S. (2015). Retos y problemáticas de la introducción de la educación mediática en los centros de secundaria. Revista de Educación, 369, 135-158.

https://doi.org/10.4438/1988-592X-RE-2015-369-293 
Murga-Menoyo, M. Á. y Novo, M. (2017). Sostenibilidad, desarrollo "glocal" y ciudadanía planetaria. Referentes de una pedagogía para el desarrollo sostenible. Teoría de la Educación, 29(1), 5578. https://doi.org/10.14201/teoredu2915579

NannieDaniel3502 (2018). The Magic School Bus So3E04 Under Construction (Structures) [Vídeo]. https://www.dailymotion.com/video/x6skmvg

Olimid, R. F. (2019). SecRet: How to apply the $5^{E}$ model for a master's level network security course. IEEE Communications Magazine, 57(11), 54-59. https://doi.org/10.1109/MCOM.001.1900228

Recomendación del Parlamento Europeo y del Consejo, de 18 de diciembre de 2006, sobre las competencias clave para el aprendizaje permanente [2006/962/CE], DOUE 394 (2006). https://eur-lex.europa.eu/legal-content/ES/TXT/?uri=celex\%3A32006Hog62

Resolución ENS/1769/2018, de 19 de julio, por la que se crea el Programa de innovación pedagógica STEAMcat y se abre convocatoria pública para la selección de centros educativos interesados en formar parte a partir del curso 2018-2019, DOGC 7671 (2018).

https://dogc.gencat.cat/es/document-del-dogc/index.html?documentId=823855 